\title{
CMEARTICLE \\ Importance of QT interval in clinical practice
}

Anand $\underline{A m b h o r e}^{1}$, MBBS, MRCP, Swee-Guan $\underline{T e O}^{2}$, MRCP, FACC, Abdul Razakjr Bin Omar $^{2}$, MRCP, FACC, Kian-Keong $\underline{P o h}^{1,3}$, FRCP, FACC

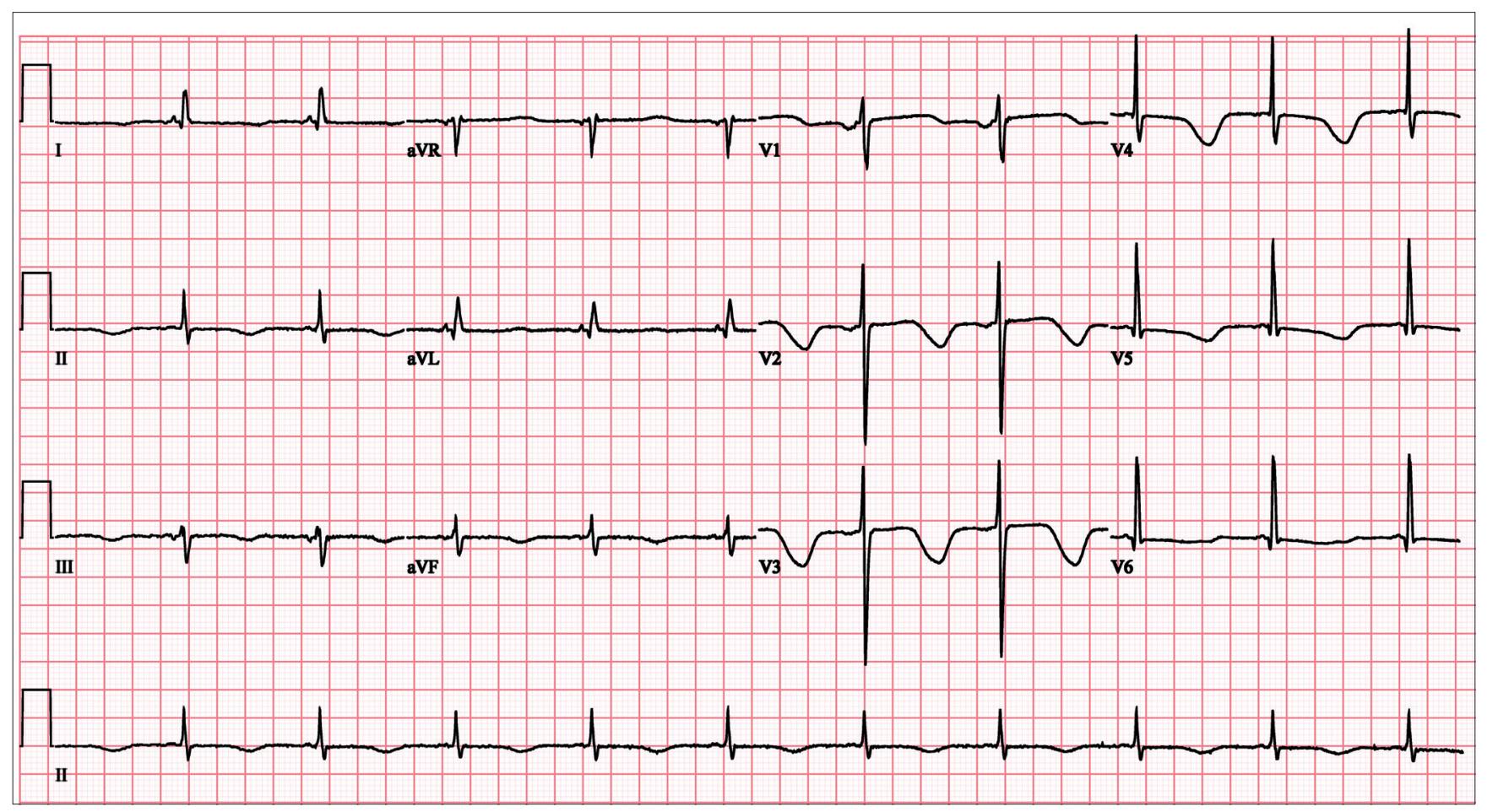

Fig. 1 Initial ECG shows sinus rhythm with deep T wave inversion and prolongation of the QTc interval.

\section{CLINICAL PRESENTATION}

A 44-year-old man presented to the emergency department with a one-week history of cough, fever, non-vertiginous giddiness and generalised weakness. He had a history of renal failure requiring renal replacement therapy, myelodysplastic syndrome and gout. One day prior to the presentation, the patient had an episode of unwitnessed seizure while resting at home. He denied any tongue-bite injury, involuntary micturition or defecation. His last dialysis was uneventful. On examination, he was found to be haemodynamically stable. On auscultation of his chest, a few right basal crepitations were heard. No focal neurological deficit was observed. The patient was admitted for suspected chest infection.

The initial laboratory evaluation showed the following: white blood cell count of $2.73 \times 10^{9} / \mathrm{L}$ with neutrophils at $61 \%$; C-reactive protein $44 \mathrm{mg} / \mathrm{L}$ (reference range $<10$ ); serum potassium $4.4 \mathrm{mmol} / \mathrm{L}$ (reference range 3.5-5.0); calcium
$2.02 \mathrm{mmol} / \mathrm{L}$ (reference range 2.15-2.55); phosphate $1.14 \mathrm{mmol} / \mathrm{L}$ (reference range 0.85-1.45); and magnesium $0.55 \mathrm{mmol} / \mathrm{L}$ (reference range 0.75-1.07). Chest radiography showed small bilateral pleural effusion with right lower lobe atelectasis, raising a suspicion of underlying pneumonia.

In the ward, the patient was febrile, although his blood cultures did not show microbial growth, he underwent treatment for chest infection with initially oral clarithromycin (one dose), which was subsequently changed to oral levofloxacin. His electrolyte imbalance was corrected. During his inpatient hospital stay, the patient developed two episodes of seizure-like events at rest, for which he underwent electroencephalography and magnetic resonance imaging of the brain, which were both normal. An electrocardiogram (ECG) was done on arrival at the emergency department.

What does the ECG in Fig. 1 show?

${ }^{1}$ Department of Cardiology, National University Heart Centre, National University Health System, ${ }^{2}$ Raffles Heart Centre, Raffles Hospital, ${ }^{3}$ Yong Loo Lin School of Medicine, National University of Singapore, Singapore

Correspondence: A/Prof Poh Kian Keong, Senior Consultant and Associate Professor, Department of Cardiology, National University Heart Centre, 1 E Kent Ridge Road, NUHS Tower Block, Level 9, Singapore 119228. kian_keong_poh@nuhs.edu.sg 


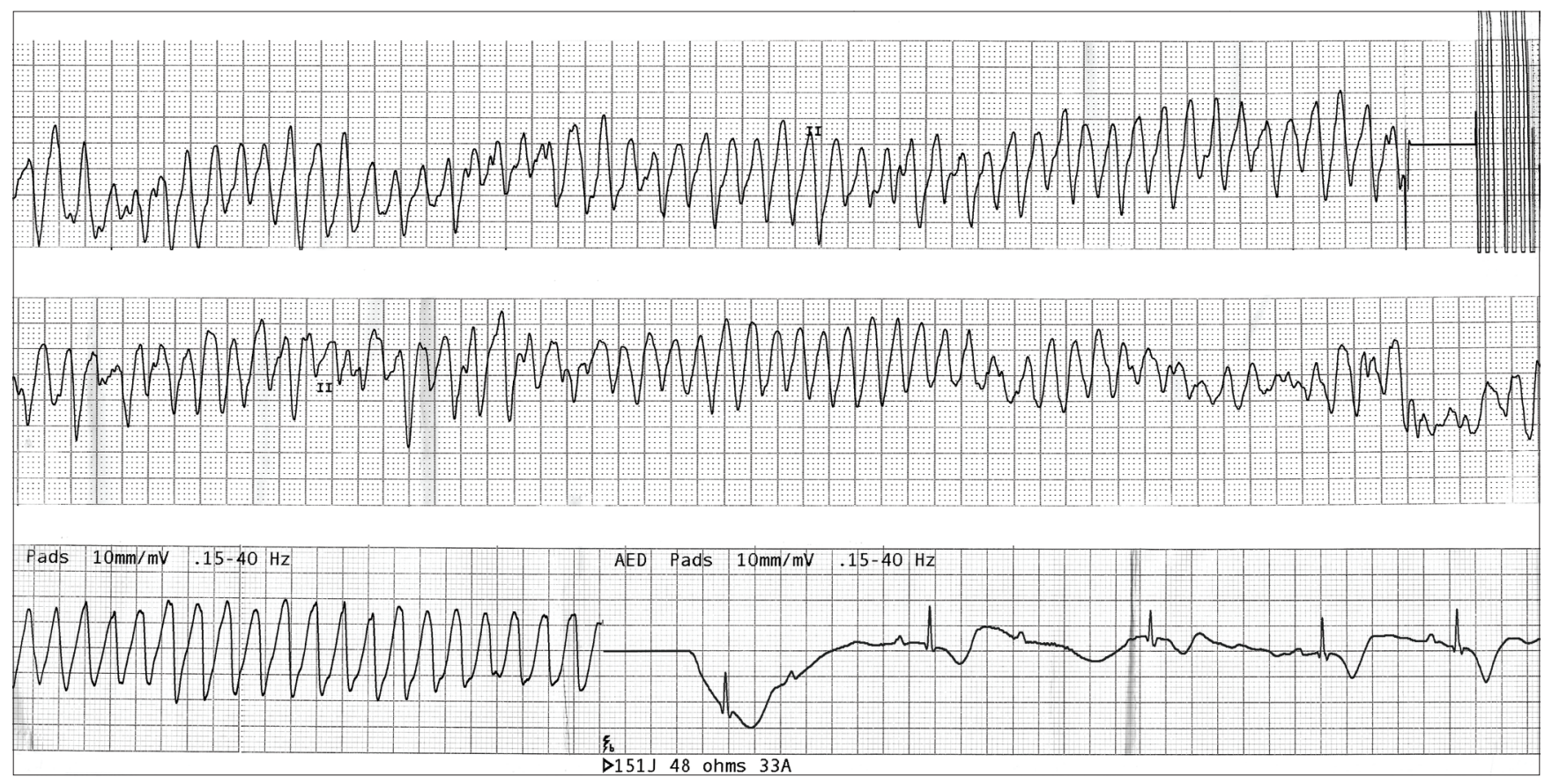

Fig. 2 ECG shows polymorphic ventricular tachycardia (torsade de pointes), which disintegrated into ventricular fibrillation, and subsequent successful electrical cardioversion.

\section{ECG INTERPRETATION}

Initial ECG (Fig. 1) shows sinus rhythm, with a heart rate of 62 beats per minute. There are deep $T$ wave inversions in the chest leads, with prolonged corrected QT interval at $700 \mathrm{msec}$. The long QT interval was presumed to be due to a combination of clarithromycin use, hypomagnesaemia and hypocalcaemia. Clarithromycin was stopped after only one dose and electrolytes were replaced. The patient was subsequently discharged well. The primary team had noticed the long QT interval and planned for 24-hour Holter monitoring, transthoracic echocardiography and subsequent cardiology review in the outpatient clinic.

\section{CLINICAL COURSE}

On the fifth day after discharge, the patient again presented to the emergency department for two brief episodes of sudden loss of consciousness, with tonic-clonic seizures and up rolling of eyes. Both episodes, which occurred after the completion of dialysis, were witnessed by the patient's wife. While waiting in the emergency department, he developed another tonic-clonic seizure. A second ECG (Fig. 2) was performed at this presentation. The ECG rhythm shows polymorphic ventricular tachycardia, with phasic variation in polarity and amplitude of QRS complexes suggestive of torsade de pointes (TdP), which subsequently disintegrated into ventricular fibrillation. A direct current shock of 150 joules converted the rhythm to sinus.

Initial electrolyte screening showed the presence of hypomagnesaemia and hypocalcaemia. The electrolytes were replaced and the patient was subsequently started on beta-blocker therapy. Transthoracic echocardiogram showed a structurally normal heart with normal left ventricular systolic function. An automatic implantable cardioverter defibrillator (AICD) was implanted before his discharge. Although genetic evaluation was not conducted, it was likely that the patient had inherited long QT syndrome (LQTS) (type III variant), based on T wave morphology in the ECG. ECG after implantation of AICD (Fig. 3) shows pacemaker rhythm (atrial-paced, ventricular-sensed). Although the QTc was still prolonged at $600 \mathrm{msec}$, it was shorter than that shown in the initial ECG (Fig 1).

\section{DISCUSSION}

LQTS due to inherited and acquired cardiac channelopathies can present with life-threatening ventricular arrhythmias in a structurally normal heart. In this case, the patient presented with symptoms of chest infection, with multiple episodes of sudden unconsciousness and seizures due to prolonged QT interval causing sustained $\mathrm{TdP}$ and ventricular arrhythmias. Prolongation of QT interval has many causes, the common ones being drugs and electrolyte disturbances. However, in this case, patient's baseline ECG prior to medication (clarithromycin and levofloxacin) showed a markedly prolonged QTC interval of 700 msec. Following correction of electrolyte abnormalities and discontinuation of the culprit medications, the patient developed two episodes of seizures due to ventricular arrhythmias, which points to a high likelihood of congenital LQTS. Hypomagnesaemia, hypocalcaemia and medications most likely unmasked the underlying condition. The patient was started on beta-blocker therapy at a maximally tolerated dose. In view of the multiple episodes of arrhythmias causing syncope, seizures and cardiac arrest, an AICD was implanted for secondary prevention.

QT interval represents the duration of ventricular electrical systole, which includes ventricular activation and recovery. It is measured from the beginning of the QRS complex to the end of the $\mathrm{T}$ wave. The duration of repolarisation (JT interval) is dependent on heart rate; QT interval is shorter with tachycardia and longer 


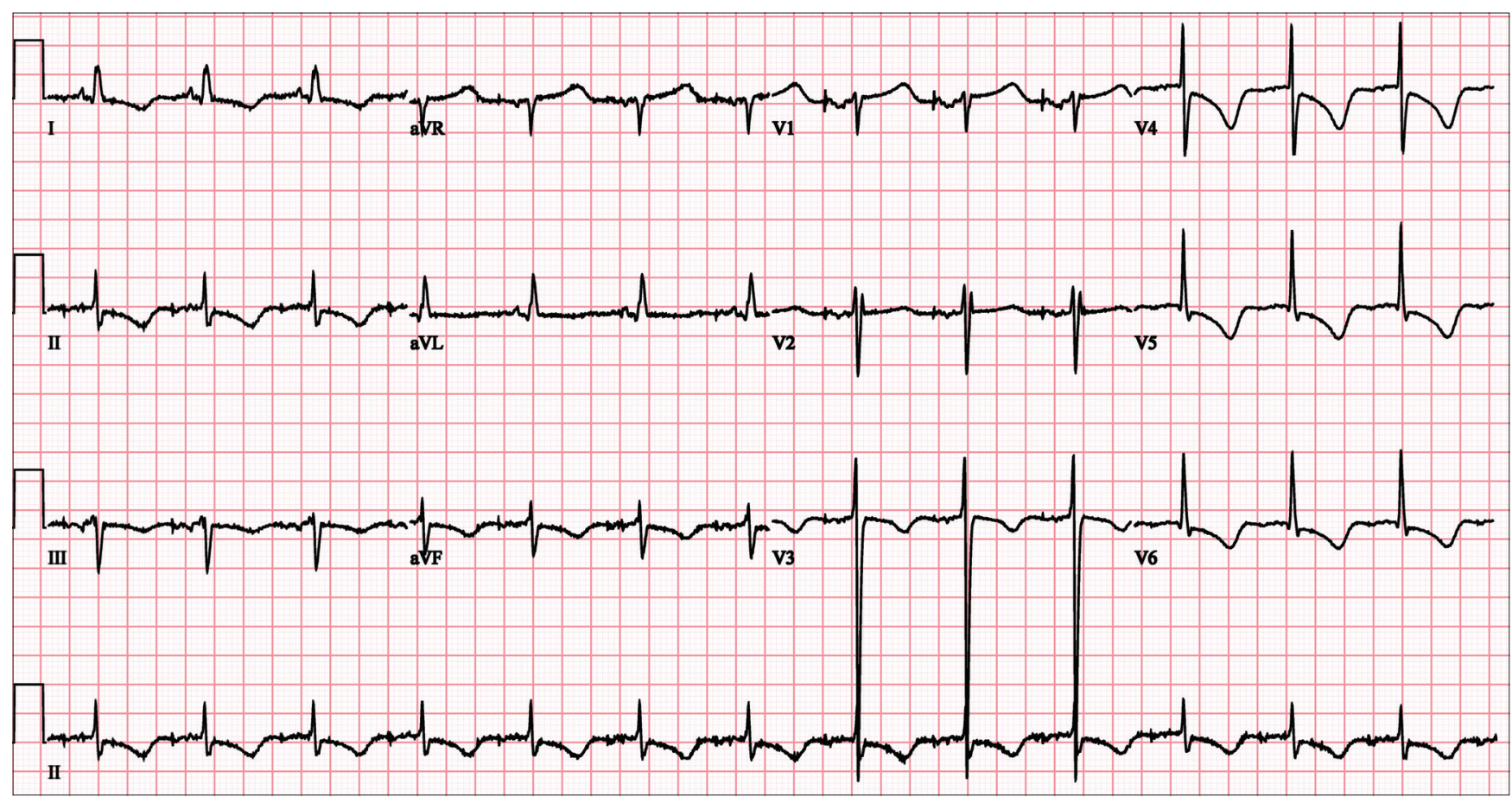

Fig. 3 Post-automatic implantable cardioverter defibrillator ECG shows pacemaker rhythm and prolonged QTc interval (600 msec).

with bradycardia. Therefore, QT interval is always corrected for the given heart rate (QTc).

There are many formulae for correction of the QT interval, including Bazett's, ${ }^{(1)}$ Fridericia's, ${ }^{(2)}$ Framingham's, ${ }^{(3)}$ normograms and linear regression formulae. In previous studies, linear regression, exponential, cubic root (Fridericia) formulae have been found to be more accurate than the Bazett's method; however, none of these are ideal even though their inaccuracy rate is in the range of $4-5$ msec, which is negligible. ${ }^{(4)}$ The most commonly used formula, the modified Bazett's formula ${ }^{(1)}$ is as follows:

$$
\mathrm{QTc}=\mathrm{QT} \text { interval } \div \sqrt{\mathrm{RR} \text { interval }(\text { in sec) }}
$$

The normal range of corrected QTc is below $0.44 \mathrm{sec}$ in males and $0.46 \mathrm{sec}$ in females; values $>0.45 \mathrm{sec}$ in males and $>0.47 \mathrm{sec}$ in females are considered as prolonged. ${ }^{(5)}$

\section{Long QT syndrome}

LQTS may be inherited or acquired, with the latter being more common. It is characterised by prolonged QT interval on ECG, which predisposes to malignant ventricular tachyarrhythmia, TdP, ventricular fibrillation and sudden death. The presentation can be variable, from asymptomatic to symptoms of syncope, seizures and palpitations to sudden death. Inherited LQTS is the prototype of 'primary cardiac arrhythmias' or 'cardiac ion channelopathies', while acquired LQTS is often a result of electrolyte disturbances and drug-induced QT prolongation.

\section{Congenital LQTS}

In 1957, Jervell and Lange-Nielsen ${ }^{(6)}$ were the first to describe a Norwegian family in which four out of ten of the children were deaf and had recurrent syncope during exercise or as a result of increased emotion. Three had died suddenly at ages four, five and nine years old. Marked QT prolongation was noted on the ECG of the children. Inheritance appeared to be autosomal recessive and associated with sensorineural deafness. ${ }^{(6)} \mathrm{A}$ similar clinical syndrome of sudden death during exercise and increased emotion, with normal hearing and autosomal dominant inheritance, was described by Romano et al in 1963 and Ward in 1964. ${ }^{(7,8)}$ The two different phenotypes of congenital LQTS were subsequently named Jervell and Lange-Nielsen syndrome for the autosomal recessive variant and Romano-Ward syndrome for the autosomal dominant variant without sensorineural deafness.

There are at least 13 LQTS genotypes (LQT 1-13) with over 200 mutations in various genes. ${ }^{(9)}$ LQTS 1, 2 and 3 are the most common, making up $80 \%-90 \%$ of the total cases of inherited LQTS. ${ }^{(9,10)}$ The three genes and related ionic currents responsible are LQTS1 (KCN 1), LQTS2 (KCNH2) and LQTS3 (SNC5A). . $^{(10)}$ Genotype is a modulator of LQTS phenotype; gene-specific differences have been described in terms of the morphology of the ST-T wave complex, triggers for cardiac events, and risk of cardiac events. ${ }^{(11)}$ Patients with LQTS1 experience the majority of events during exercise or heightened emotions, those with LQTS2 during heightened emotion and those with LQTS3 usually during sleep and less with exercise. ${ }^{(10)}$

Although the most important expected finding in these cases is the presence of prolonged QT interval, up to $10 \%-35 \%$ of patients with LQTS may present with a normal QT interval, ${ }^{(12)}$ which makes it difficult to screen such patients. The T wave morphology may give some clue regarding the type of LQTS. LQT 1 usually has an infantile ST-T wave pattern, with broad pronounced T wave, LQT 2 commonly has low-amplitude bifid T wave, and LQT 3 has late-appearing $T$ wave with steep downslope. ${ }^{(13)}$

Depending on the genotypes, QTc values > 500 msec confer a five- to eightfold increased risk of experiencing at least one 
cardiac event up to the age of 40 years. ${ }^{(11)}$ Highest-risk patients for cardiac events before 40 years of age comprise females with LQTS2, and males with LQTS3 and QTc > 500 msec. ${ }^{(11)}$ These two genotypes also have a suboptimal response to beta-blocker therapy, unlike LQTS1.(14)

Management of patients with inherited LQTS include: (a) avoidance of triggers such as exercise, competitive sports (ACC/AHA class I indication) and drugs that prolong QT interval; (b) prevention of potassium loss and prompt replacement of any loss; and (c) use of appropriate beta-blocker therapy. Beta-blockers are the mainstay of therapy, with propranolol (2-4 mg/kg/day), metoprolol (2-4 mg/kg/day) and nadolol (1-2.5 mg/kg/day) being most commonly administered. The ACC/AHA/ESC guidelines on prevention of sudden cardiac death indicate that ICD is a class I indication in all patients with LQTS with prior cardiac arrest, and a class IIb indication in the presence of QTc > 500 msec LQTS 2 and LQTS 3 subtypes, which are less responsive to conventional beta-blocker therapy. ${ }^{(15)}$

\section{Acquired LQTS}

The main predisposing factors for acquired LQTS are mediations and electrolyte imbalances, commonly hypomagnesaemia, hypokalaemia or hypocalcaemia. ${ }^{(16)}$ Additionally conditions like extreme bradycardia due to atrioventricular nodal disease or sick sinus syndrome, hypothyroidism, hypothermia and anorexia nervosa can predispose patients to prolonged QT interval. It must be kept in mind that drugs are the most common causes of prolonged QT interval. In the past, several drugs have been withdrawn or received black box warning due to their potential to cause QT interval prolongation that may lead to fatal ventricular arrhythmias and sudden cardiac deaths. ${ }^{(16,17)}$

\section{Torsade de pointes}

$\mathrm{TdP}$ is a type of polymorphic ventricular tachycardia associated with prolonged QT interval or increased $U$ wave amplitude that responds to increases in the heart rate. ${ }^{(18,19)}$ To be considered TdP, the axis of the QRS complex must change in direction and show variation in polarity and amplitude. ${ }^{(20)}$ Tachycardia is usually at the rate of 200-250 beats per minute. Although not pathognomonic, in most cases, it is preceded by a sequence of a long RR interval of the dominant cycle followed a short extrasystolic interval with premature depolarisation interrupting the T wave. ${ }^{(21)}$

Some of commonly prescribed drugs that can result in prolonged QT interval include: ${ }^{(16,17,22-25)}$ (a) antiarrhythmic agents: procainamide, quinidine, amiodarone, flecainide, propofenone, disopyramide, dronedarone, sotalol, dofetilide, ibutilide; (b) antihistamines: terfenadine, astimazole; (c) antipsychotics: quetiapine, haloperidol, clozapine; (d) tri- and tetra-cyclic antidepressants: imipramine, doxepin; (e) selective serotonin reuptake inhibitors: citalopram, fluoxetine; (f) antimicrobial agents: fluoroquinolones (ciprofloxacin, levofloxacin, moxifloxacin, gatifloxacin, ofloxacin), macrolides (erythromycin, clarithromycin, azithromycin), antifungal agents (ketokonazole, voriconazole); and (g) antimalarial agents: quinine, mefloquine, chloroquine.
Currently, more than 50 commonly prescribed medications cause QT prolongation and/or have been associated with TdP or sudden death. Management of acquired LQTS involves the correction of electrolyte imbalances, avoidance of medications that can cause prolong QT and TdP, ECG monitoring and prompt treatment of arrhyrhmias. In patients with TdP, prompt electric defibrillation should be administered in haemodynamically unstable patients. Intravenous magnesium sulphate is highly effective in the treatment and prevention of long QT-related arrhythmias. ${ }^{(26,27)}$ Other therapies include overdrive pacing and the use of isoproterenol. ${ }^{(26)}$

In conclusion, the finding of long QT interval is important in clinical practice. However, this is often missed when interpreting ECG. Inherited and acquired LQTS is a potentially lethal cardiac channelopathy that is frequently mistaken for epilepsy. As many drugs can cause prolongation of the QT interval, physicians should be cognizant of this fact when prescribing such drugs to at-risk patients.

ABSTRACT Long QT interval is an important finding that is often missed by electrocardiogram interpreters. Long QT syndrome (inherited and acquired) is a potentially lethal cardiac channelopathy that is frequently mistaken for epilepsy. We present a case of long QT syndrome with multiple cardiac arrests presenting as syncope and seizures. The long QTc interval was aggravated by hypomagnesaemia and drugs, including clarithromycin and levofloxacin. Multiple drugs can cause prolongation of the QT interval, and all physicians should bear this in mind when prescribing these drugs.

Keywords: causes, diagnosis and management, ECG, long QT interval, torsade de pointes

\section{REFERENCES}

1. Bazett HC. An analysis of time relations of the electrocardiogram. Heart 1920; 7:353-70

2. Fridericia LS. The duration of systole in an electrocardiogram in normal humans and in patients with heart disease. 1920. Ann Noninvasive Electrocardiol 2003; 8:343-51.

3. Sagie A, Larson MG, Goldberg RJ, Bengtson JR, Levy D. An improved method for adjusting the QT interval for heart rate (the Framingham Heart Study). Am J Cardiol 1992; 70:797-801.

4. Malik M. Problems of heart rate correction in assessment of drug-induced QT prolongation interval. J Cardiovasc Electrophysiol 2001; 12:411-20.

5. Moss AJ. Measurement of the QT interval and the risk associated with QTC interval prolongation: a review. Am J Cardiol 1993; 72:23B-25B.

6. Jervell A, Lange-Nielsen F. Congenital deaf-mutism, functional heart disease with prolongation of the QT interval and sudden death. Am Heart J 1957; 54:59-68.

7. Romano C, Gemmer G, Pongiglione R. [Rare cardiac arrythmias of the pediatric age. II. Syncopal attacks due to paroxysmal ventricular fibrillation (presentation of 1 st case in italian pediatric literature)]. Clin Pediatr 1963; 45:656-83. Italian.

8. Ward OC: A new familial cardiac syndrome in children. J Ir Med Assoc 1964; 54:103-106.

9. Splawski I, Shen J, Timothy KW, et al. Spectrum of mutations in longqt syndrome genes. KVLQT1, HERG, SCN5A, KCNE1, and KCNE2. Circulation 2000; 102:1178-85.

10. Schwartz PJ, Priori SG, Spazzolini C, et al. Genotype-phenotype correlation in the long-QT syndrome: gene-specific triggers for life-threatening arrhythmias. Circulation 2001; 103:89-95

11. Priori SG, Schwartz PJ, Napolitano C, et al. Risk stratification in the longQT syndrome. N Engl J Med 2003; 348:1866-74.

12. Napolitano C, Priori SG, Schwartz PJ, et al. Genetic testing in the long 
QT syndrome: development and validation of an efficient approach to genotyping in clinical practice. JAMA 2005; 294: 2975-80.

13. Zhang L, Timothy KW, Vincent GM, et al. Spectrum of ST-T-wave patterns and repolarization parameters in congenital long-QT syndrome: ECG findings identify genotypes. Circulation 2000; 102:2849-55.

14. Priori SG, Napolitano C, Schwartz PJ, et al. Association of long QT syndrome loci and cardiac events among patients treated with betablockers. JAMA 2004; 292:1341-4.

15. Zipes DP, Camm AJ, Borggrefe M, et al. ACC/AHA/ESC 2006 guidelines for management of patients with ventricular arrhythmias and the prevention of sudden cardiac death: a report of the American College of Cardiology/ American Heart Association Task force and the European Society of Cardiology Committee for practice guidelines. J Am Coll Cardiol 2006; 48: e247-346.

16. Al-Khatib SM, Allen LaPionte N, Kramer JM, Califf RM. What clinicians should know about the QT interval? JAMA 2003; 289:2120-7.

17. Yap YG, Camm AJ. Drug induced QT prolongation and torsades de pointes. Heart 2003; 89:1363-72.

18. Coumel P, Leclercq JF, Lucet V. Possible mechanisms of the arrhythmias in long QT syndrome. Eur Heart J 1985; 6 Suppl D:115-29.

19. Nguyen PT, Scheinman MM, Sege J. Polymorphous ventricular tachycardia: clinical characterization, therapy and the QT interval. Circulation 1986; 74:340-9.

20. Sclarovsky S, Strasberg B, Lewin RF, Agmon J. Polymorphous ventricular tachycardia: clinical features and treatment. Am J Cardiol 1979; 44:339-44.

21. Kay GN, Plumb VJ, Arciniegas JG, Henthorn RW, Waldo AL. Torsade de pointes: the long-short initiating sequence and other clinical features: observations in 32 patients. J Am Coll Cardiol 1983; 2:806-17.

22. Darpo B. Spectrum of drugs prolonging QT interval and the incidence of torsades de pointes. Eur Heart J Supplements 2001; 3(suppl K):S70-S80.

23. Taylor DM. Antipsychotics and QT prolongation. Acta Psychiatr Scand 2003; 107:85-95.

24. Haddad PM, Anderson IM. Anti-psychotic related QTc prolongation, torsades de pointes and sudden death. Drugs 2002; 62:1649-71.

25. Woosley RL. Drugs that prolong the QT interval and/or induce torsades de pointes. Revised November 18, 2014 [online]. Available at: www. QTdrugs.org. Accessed November 30, 2014.

26. Khan IA. Long QT syndrome: diagnosis and management. Am Heart J 2002; 143:7-14

27. Tzivoni D, Banai S, Schuger C, et al. Treatment of torsade de pointes with magnesium sulfate. Circulation 1988; 77:392-7. 


\section{SINGAPORE MEDICAL COUNCIL CATEGORY 3B CME PROGRAMME} (Code SMJ 201412A)

Question 1. The following medications may result in prolongation of QT interval:
(a) Tricyclic antidepressant.
(b) Penicillin.
(c) The antihistamine, terfenadine.
(d) Clarithromycin.

Question 2. Regarding torsade de pointes:

(a) Hypomagnesaemia may be a triggering factor.

(b) Tachycardia may abort it.

(c) It is a type of monomorphic ventricular tachycardia.

(d) Amiodarone is the drug of choice.

Question 3. The following electrolyte abnormalities may cause prolongation of QT interval:
(a) Hypercalcaemia.
(b) Hypomagnesia.
(c) Hypokalaemia.
(d) Hypophosphataemia.

Question 4. Regarding QT interval:

(a) It represents ventricular electrical diastole.

(b) Tachycardia tends to shorten QT interval.

(c) Sleep causes prolongation of QT interval.

(d) Studies have found linear regression formulae to be better than the Bazett's formula for correction of QT interval.

Question 5. Regarding congenital long QT syndrome (LQTS):

(a) It always has marked prolonged QT interval.

(b) Beta-blockers are the drug of choice, especially for LQTS1.

(c) Poor response to beta-blockers and LQTS 2/3 subtypes results in a higher risk of sudden death.

(d) The use of an implantable cardioverter defibrillator is indicated in all patients of previous cardiac arrest.

\section{Doctor's particulars:}

Name in full

MCR number

Email address

\section{SUBMISSION INSTRUCTIONS:}

(1) Log on at the SMJ website: http://www.sma.org.sg/publications/smjcurrentissue.aspx and select the appropriate set of questions. (2) Provide your name, email address and MCR number. (3) Select your answers and click "Submit".

\section{RESULTS:}

(1) Answers will be published in the SMJ February 2015 issue. (2) The MCR numbers of successful candidates will be posted online at the SMJ website by 30 January 2015 (3) Passing mark is $60 \%$. No mark will be deducted for incorrect answers. (4) The SMJ editorial office will submit the list of successful candidates to the Singapore Medical Council. (5) One CME point is awarded for successful candidates.

Deadline for submission: (December 2014 SMJ 3B CME programme): 12 noon, 23 January 2015. 